\title{
In-situ electrical measurements of vertically aligned nanostructures
}

\author{
G. McMahon,* T. Paudel,* Z.F. Ren, and M.J. Naughton* \\ * Department of Physics, Boston College, Chestnut Hill, MA 02467
}

We have initiated a major thrust towards integrated sciences at Boston College, a major component of which included the construction of a 1,500 square foot class 10,000/1,000 clean room facility housing, among other deposition and characterization equipment, a JEOL 7001F SEM with a Nabity system for e-beam lithography, and a JEOL 4500 Multibeam instrument equipped with four Kleindiek nanomanipulators with low noise triaxial electrical connections to a Keithley 2612A sourcemeter.

A large component of our research involves the fabrication of carbon nanotubes and nanopillar structures for a variety of applications, including solar cell and biological sensors. Necessary towards our research are electrical measurements of these structures on an individual, rather than aggregate, basis.

There are several examples in the literature of electrical measurements of single carbon nanotubes. These include measurements made in-situ in a TEM using a specialized holder [1], and others where the nanotubes were manipulated onto prefabricated metal electrodes [2]. Both of these methodologies are very time consuming and necessarily separate the structure under test from the final device.

By incorporating the four point probe station into the Multibeam system, we can not only perform electrical measurements on individual nanotubes or nanopillar structures, but can also use the gas deposition capabilities of the FIB to deposit carbon nanostructures using either the electron or ion beam. This allows us to measure the electrical properties of these structures in-situ without ever breaking vacuum. The FIB offers an additional capability: we can use it to sputter-clean the W probe tips after an accumulation of carbonaceous material.

An example of the measurement of a carbon nanotube deposited as part of an array fabricated using e-beam lithography is shown in Fig. 1. For this measurement, we swept a variable voltage between the nanotube and the substrate, and then measured the current flowing through the nanotube itself. The measurement was repeated 10 times. The results are given in Fig. 2. We have just begun the interpretation of the results, in particular the shift of the curve towards higher measured currents after the first three measurements. Further tests are required to determine if this is a result of a change in the contact between the probe and the carbon nanotube, or a change in the structure and electrical properties of the carbon nanotube initiated by localized resistive heating during the course of the first few measurements.

References

[1] J.Y. Huang et al., Phys. Rev. Lett. 94 (2005) 236802.

[2] H. Dai, E.W. Wong and C.M. Lieber, Science (272) (1996) 523. 


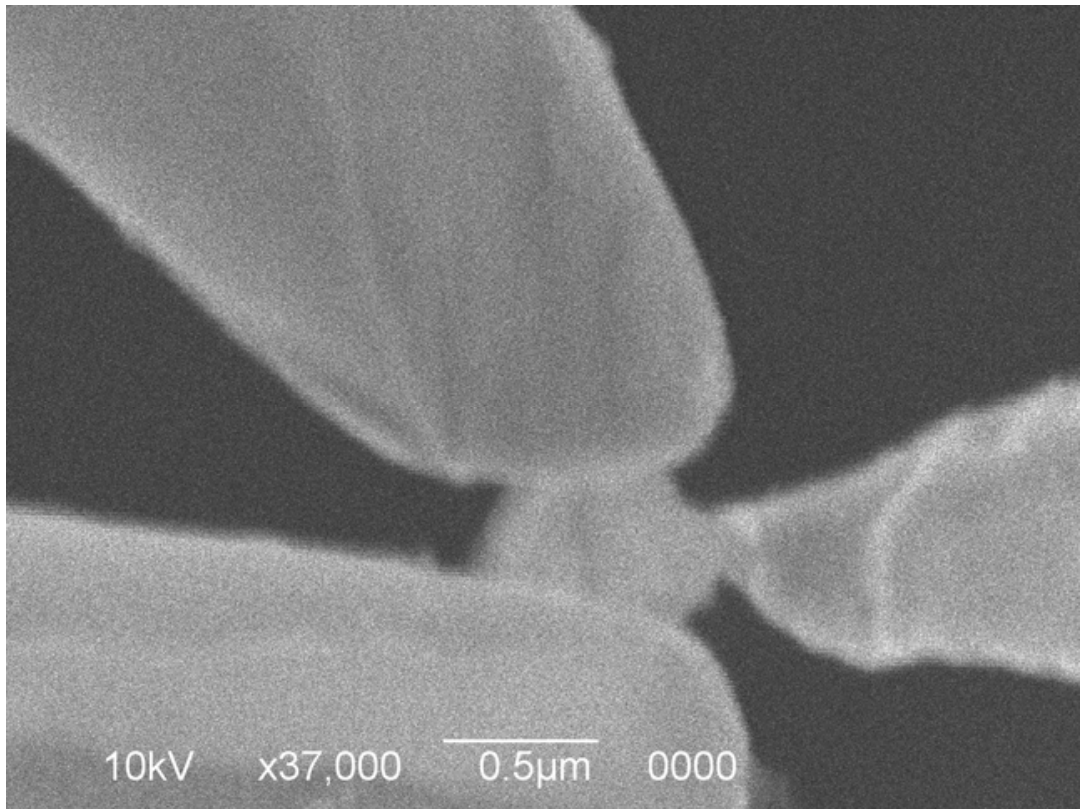

Figure 1: Three W probe contacts to a carbon nanotube (source lo, sense lo and sense hi). Probes were brought to contact the substrate, then raised until they were no longer contacting the substrate, and brought to contact the CNT. The CNT was surprisingly stiff, such that when the tips came into contact, they would slide along it rather than push it, making identification of the point of contact much easier.

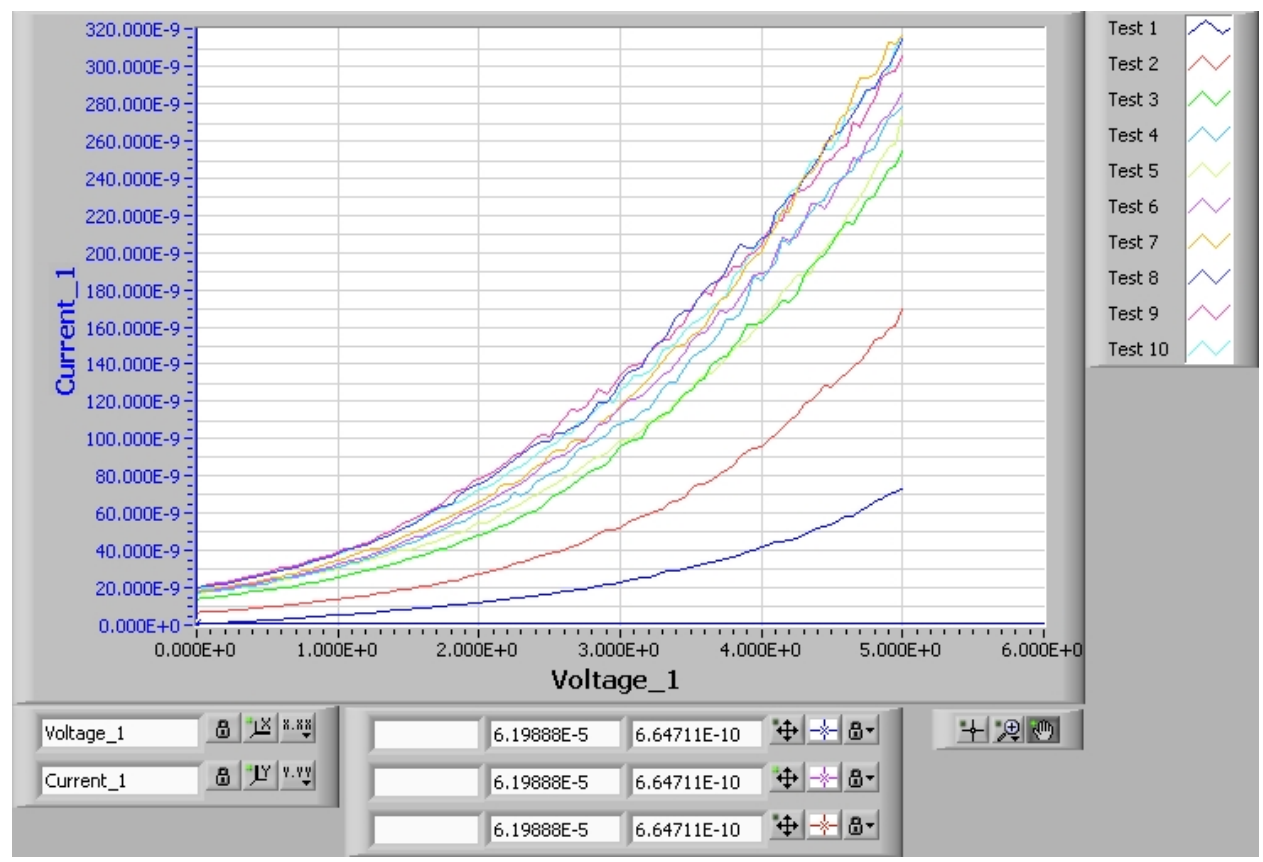

Figure 2: Results of four point electrical measurement on carbon nanotube (CNT) shown above. Voltage swept from $0-5$ volts between the CNT and substrate. The current is measured on the CNT. 100 data points. 10 consecutive tests. Note shift towards higher current after tests 1 (blue) and test 2 (red). 